\title{
13. Qualifizierung im freiwilligen Engagement
}

\author{
Julia Simonson \& Laura Romeu Gordo
}

\section{Kernaussagen}

Für freiwillige Tätigkeiten sind häufig spezifische Qualifikationen nötig. Fast ein Viertel der Engagierten übt eine Tätigkeit aus, für die eine spezifische Aus- oder Weiterbildung erforderlich ist. Dabei üben Männer deutlich häufiger als Frauen eine Tätigkeit aus, die eine spezifische Qualifikation erfordert. Die Qualifikationsanforderungen variieren darüber hinaus zwischen den gesellschaftlichen Bereichen. Am häufigsten benötigen die Engagierten im Bereich der Unfall-und Rettungsdienste eine spezielle Aus- oder Weiterbildung.

Viele Engagierte nehmen im Rahmen ihres Engagements an Weiterbildungen teil. Zwei Fünftel aller Engagierten haben im Rahmen ihres Engagements schon einmal oder mehrmals an einer Weiterbildung teilgenommen, Männer dabei zu größeren Anteilen als Frauen. Personen mit niedriger Bildung nehmen zu ähnlichen Anteilen an Weiterbildungen im Engagement teil wie Personen mit mittlerer oder höherer Bildung.

Ein Großteil der Engagierten gibt an, im Engagement dazu zu lernen. Knapp drei Viertel der Engagierten geben an, soziale Fähigkeiten erlangt zu haben. Jeweils mehr als die Hälfte der Engagierten hat aber auch persönliche Fähigkeiten oder Fachkenntnisse im Engagement erworben. Dabei geben Männer deutlich häufiger als Frauen an, Fachkenntnisse hinzugewonnen zu haben. Schülerinnen und Schüler erwerben besonders häufig soziale und persönliche Fähigkeiten im Engagement.

Die Engagierten erachten im Engagement erworbene Fähigkeiten auch für andere Lebensbereiche von Nutzen. Ein Großteil der Engagierten bewertet die erworbenen Kenntnisse und Fähigkeiten als nützlich für Schule, Ausbildung oder Studium, die berufliche Tätigkeit oder für den beruflichen Wiedereinstieg. Dabei profitieren Engagierte mit höherer Bildung am meisten von den erworbenen Fähigkeiten. Sie berichten häufiger als Engagierte mit niedriger oder mittlerer Bildung, dass die im Engagement erworbenen Fähigkeiten für den beruflichen Wiedereinstieg oder für die berufliche Tätigkeit von Nutzen sind.

\subsection{Einleitung}

Freiwilliges Engagement bietet Möglichkeiten für informelles und formelles Lernen und ermöglicht es, Kompetenzen personaler, sozialer, kultureller sowie instrumenteller Art zu erwerben (Düx, Prein, Sass \& Tully 2009). Freiwillige Tätigkeiten unterscheiden sich hinsichtlich der notwendigen und vorausgesetzten oder $\mathrm{zu}$ erwerbenden Kenntnisse und Fähigkeiten. Wäh- rend für manche Tätigkeiten keine oder nur sehr allgemeine Vorkenntnisse erforderlich sind, benötigen andere Einsatzfelder sehr spezifische Kenntnisse und Fertigkeiten. Spezielle Tätigkeiten, zum Beispiel im Rettungsdienst oder auch in der Telefonseelsorge, lassen sich ohne fundierte Ausbildung nicht ausüben. Maßnahmen der Qualifizierung für Engagierte können daher

(C) Der/die Autor(en) 2017

J. Simonson et al. (Hrsg.), Freiwilliges Engagement in

Deutschland, Empirische Studien zum bürgerschaftlichen

Engagement, DOI 10.1007/978-3-658-12644-5_14 
in verschiedenen Formen und mit unterschiedlichen Zielstellungen und Inhalten angeboten werden (Hartnuß \& Kegel 2011).

Qualifizierung im Engagement ist kein neues Phänomen. Die Anforderung zur Vorbereitung und Weiterbildung war in bestimmten Bereichen wie den Freiwilligen Feuerwehren oder den Rettungsdiensten schon immer wichtig (Jakob 2013). Allerdings ändern sich die freiwilligen Tätigkeiten und es gibt mehr neue und komplexe Aufgaben, die die Anforderungen zur Qualifizierung erhöhen. Nach der Enquete-Kommission ,Zukunft des Bürgerschaftlichen Engagements (2002) beobachten wir eine Pluralisierung des bürgerschaftlichen Engagements - die Betätigungsfelder werden vielfältiger und die Tätigkeitsdichte steigt (siehe Kapitel 11).

Für die Engagierten können Qualifizierungschancen einen Anreiz bieten, freiwilliges Engagement aufzunehmen oder fortzuführen. Qualifizierungsangebote können dabei als Subventionen verstanden werden, die die individuellen Kosten, die sonst für eine Weiterbildung anfallen würden, mindern (Mildenberger 2012: 176f.). Im Idealfall können die im Rahmen des Engagements erworbenen Kenntnisse nicht nur in der freiwilligen Tätigkeit, sondern auch in anderen Kontexten wie zum Beispiel im Beruf nützlich sein. Darüber hinaus kann die Möglichkeit sich weiterzubilden, eine Anerkennung für die freiwillige Tätigkeit sein. Befunde des Freiwilligensurveys zeigen, dass die Erwartung, Kenntnisse und Erfahrungen erweitern zu können, ein zentrales Motiv für freiwilliges Engagement ist (Gensicke 2015; siehe auch Kapitel 15). Außerdem sind dabei auch die Möglichkeiten für den informellen Erfahrungsaustausch zwischen den Engagierten bedeutsam (Jakob 2013). Wichtig für Qualifizierungsangebote sind auch die Kenntnisse und Erfahrungen, die die Engagierten aus anderen Bereichen in die Organisationen einbringen können. Qualifizierungsmaßnahmen sollten diesen Sachverhalt berücksichtigen und die Angebote an die vorhandenen Kompetenzen anpassen.
Häufig wird das Thema Kompetenzerwerb und Lernen im freiwilligen Engagement vor allem mit Bezug auf Jugendliche oder jüngere Erwachsene betrachtet (so z. B. bei Düx 2006; Prein, Sass \& Züchner 2009). Da Lernen grundsätzlich bis ins hohe Erwachsenenalter hinein stattfinden kann (Leipold 2012; Reich-Claassen \& Tippelt 2013), stellen sich Fragen der Weiterbildung im Engagement jedoch auch für höhere Altersgruppen. In diesem Kapitel werden Weiterbildung und Kompetenzerwerb im freiwilligen Engagement daher für alle Altersgruppen betrachtet.

Auf Basis des Deutschen Alterssurveys (DEAS) lässt sich zeigen, dass Frauen, Ältere und Geringergebildete seltener Bildungsaktivitäten ausüben (Naumann \& Romeu Gordo 2010; Simonson, Hagen, Vogel \& Motel-Klingebiel 2013). Ungleiche Zugänge zu Bildungsmaßnahmen fanden sich in der Vergangenheit auch in der Qualifizierung im und für das Engagement. Der Freiwilligensurvey 2009 zeigte, dass Frauen, Arbeitslose und Personen aus den neuen Bundesländern unterdurchschnittlich häufig an Qualifizierungsmaßnahmen im Rahmen ihres Engagements teilnehmen (Gensicke 2015). Problematisch kann dies werden, wenn aus den unterschiedlichen Qualifizierungsbeteiligungen ungleiche Chancen, sich im Engagement zu beteiligen, erwachsen. In diesem Kapitel soll gezielt der Frage nachgegangen werden, wie sich Weiterbildung im Engagement zwischen Frauen und Männern unterscheidet und inwieweit sich darüber hinaus ungleiche Zugangsmuster nach Alters- und Bildungsgruppen zeigen lassen.

In diesem Kapitel soll konkret folgenden Forschungsfragen nachgegangen werden:

1. Inwieweit wird für die Ausübung des Engagements eine spezielle Aus- und Weiterbildung vorausgesetzt? Wie unterscheidet sich dies nach Geschlecht, Alters- und Bildungsgruppen sowie nach Engagementbereichen?

2. Wie verbreitet sind Qualifizierungsmaßnahmen im Engagement und von wem werden die Qualifizierungsangebote genutzt? Gibt es 
Unterschiede zwischen Frauen und Männern, zwischen Alters- und Bildungsgruppen?

3. Welche Kenntnisse werden im Engagement erworben und inwieweit sind diese Kennt- nisse auch im schulischen und beruflichen Bereich von Nutzen? Profitieren Frauen und Männer sowie verschiedene Bildungsgruppen in unterschiedlichem Ausmaß von den im Engagement erworbenen Kenntnissen?

\subsection{Datengrundlage}

Die in diesem Kapitel berichteten Befunde basieren auf gewichteten Analysen. Für die Gewichtung wurden in allen Erhebungsjahren dieselben Gewichtungsmerkmale berücksichtigt. Dabei handelt es sich um die Designgewichtung, die sich auf die Auswahlwahrscheinlichkeit einer Person bezieht (Haushaltsgröße, Zahl der Festnetznummern und, nur für das Jahr 2014, auch die Zahl der Mobilfunknummern) und Gewichtungsmerkmale, die Abweichungen der Stichprobenverteilung von der Grundgesamtheit in bestimmten Dimensionen ausgleichen (Bundesland, Gemeindegrößenklassen, Geschlecht, Altersgruppen). Von einer Hinzunahme weiterer Gewichtungsmerkmale wie etwa des Bildungsstandes haben wir abgesehen, um die Vergleichbarkeit mit den bisher veröffentlichten Berichten zu erhalten.

Wir untersuchen Qualifikationsanforderungen im freiwilligen Engagement, die Teilnahme an Qualifizierungsmaßnahmen sowie im Rahmen des Engagements erworbene Fähigkeiten und ihre Nützlichkeit für die Engagierten. Diese Aspekte werden im Freiwilligensurvey für die zeitintensivste Tätigkeit derjenigen Engagierten erfasst, die mehr als eine Tätigkeit ausüben, beziehungsweise bei Personen mit einer freiwilligen Tätigkeit für diese. Alle Angaben zu Qualifikationsanforderungen, Qualifizierungsmaßnahmen und erworbenen Fähigkeiten sind selbst berichtet.

Im Freiwilligensurvey 2014 wird folgendermaßen zu Qualifikationsanforderungen gefragt:

Ist für Ihre Tätigkeit eine spezifische Ausbildung oder Weiterbildung erforderlich?
Antwortkategorien: ja; nein

Darüber hinaus wird auch nach der Teilnahme an Qualifikationsmaßnahmen im Rahmen des Engagements gefragt:

Haben Sie im Zusammenhang mit Ihrer Tätigkeit schon einmal an Kursen oder Seminaren zur Weiterbildung teilgenommen?

Die Antwortkategorien für diese Frage sind ,ja, einmal', ,ja, mehrmals' und ,nein.

Es wird auch nach im Rahmen des Engagements erworbenen Fähigkeiten gefragt. Dabei werden drei verschiedene Kategorien von Fähigkeiten unterschieden: Fachkenntnisse, soziale Fähigkeiten und persönliche Fähigkeiten.

Haben Sie im Rahmen Ihrer Tätigkeit folgende Fähigkeiten oder Kenntnisse erworben?

\section{Fachkenntnisse?}

Soziale Fähigkeiten, zum Beispiel Teamfähigkeit, Zuhören können?

Persönliche Fähigkeiten, zum Beispiel Selbstständigkeit, Zeitmanagement, Arbeitstechniken?

Die Antwortkategorien für diese Frage sind für jede Art von Fähigkeiten ,ja und ,nein'. Mehrfachnennungen sind möglich.

Bei Engagierten, die über erworbene Fähigkeiten berichten, wird die Nützlichkeit dieser 
Fähigkeiten in unterschiedlichen Bereichen abgefragt:

Sind diese Fähigkeiten und Kenntnisse in dem/n folgenden Bereich/en nützlich?

In der Schule, Ausbildung oder Studium (nur an Personen in Schule, Ausbildung oder Studium)?

Beim Wiedereinstieg in den Beruf (nur an Personen die arbeitslos, in Mutterschutz, Elternzeit, in Umschulung/Weiterbildung oder seit weniger als einem Jahr erwerbstätig sind)?

Bei der beruflichen Tätigkeit (nur an Erwerbstätige)?
Die Antwortkategorien für diese Frage sind jeweils ,ja und ,nein`.

In den Analysen haben wir Gruppenunterschiede etwa zwischen Frauen und Männern oder zwischen Personen mit niedriger Bildung und Personen mit hoher Bildung auf statistische Signifikanz getestet (siehe Kapitel 2.7). Ist ein Befund statistisch signifikant, so kann dieses Ergebnis auf die Grundgesamtheit der Wohnbevölkerung Deutschlands ab 14 Jahren verallgemeinert werden. Der Begriff ,statistisch signifikant' bedeutet jedoch nicht, dass ein Ergebnis wichtiger oder bedeutsamer als ein anderes Ergebnis ist.

\subsection{Qualifikationsvoraussetzungen im Engagement}

Im Folgenden wird zunächst der Frage nachgegangen, inwieweit eine spezielle Aus- und Weiterbildung notwendig ist, um sich freiwillig $\mathrm{zu}$ engagieren, und wie sich diese Qualifikationsanforderungen nach Geschlecht, Alter und Bildungsgruppen sowie nach Engagementbereichen unterscheiden.

Die in Abbildung 13-1a dargestellten Ergebnisse zeigen, dass mit 22,6 Prozent fast ein Viertel der Engagierten eine Tätigkeit ausübt, für die eine spezifische Aus- oder Weiterbildung erforderlich ist. Die Qualifikationsanforderungen unterscheiden sich dabei signifikant zwischen Frauen und Männern. Während 27,5 Prozent der engagierten Männer eine spezifische Qualifikation für ihr Engagement benötigen, ist das nur für 17,4 Prozent der engagierten Frauen der Fall. Dies könnte zum einen darauf zurückzuführen sein, dass Männer sich häufiger in gesellschaftlichen Bereichen engagieren, in denen spezifische Qualifikationen benötigt werden, beispielsweise im Bereich der Unfall- oder Rettungsdienste. Zum anderen könnte es auch damit zusammenhängen, dass Frauen im Rahmen ihres freiwilligen Engagements seltener eine Leitungs- oder Vorstandstätigkeit ausüben, für die spezifische Qualifikationen notwendig sind.

Nach Altersgruppen betrachtet, lassen sich nur geringe Unterschiede hinsichtlich der Qualifikationsanforderungen finden. Engagierte der Altersgruppe 65 Jahre und älter üben dabei am seltensten eine Tätigkeit aus, für die eine spezielle Aus- oder Weiterbildung notwendig ist (21,2 Prozent).

In Abbildung 13-1b wird deutlich, dass Engagierte mit hoher Bildung öfter eine Tätigkeit ausüben, für die eine spezifische Ausbildung oder Weiterbildung erforderlich ist (24,9 Prozent), als Engagierte anderer Bildungsgruppen. Die übrigen Bildungsgruppen unterscheiden sich dagegen in dieser Hinsicht nicht signifikant voneinander. 
Abbildung 13-1: Anteile der Engagierten, für deren freiwillige Tätigkeit eine spezifische Aus- oder Weiterbildung erforderlich ist, 2014, a) gesamt, nach Geschlecht und nach Alter, b) nach Bildung

a) gesamt, nach Geschlecht und nach Alter

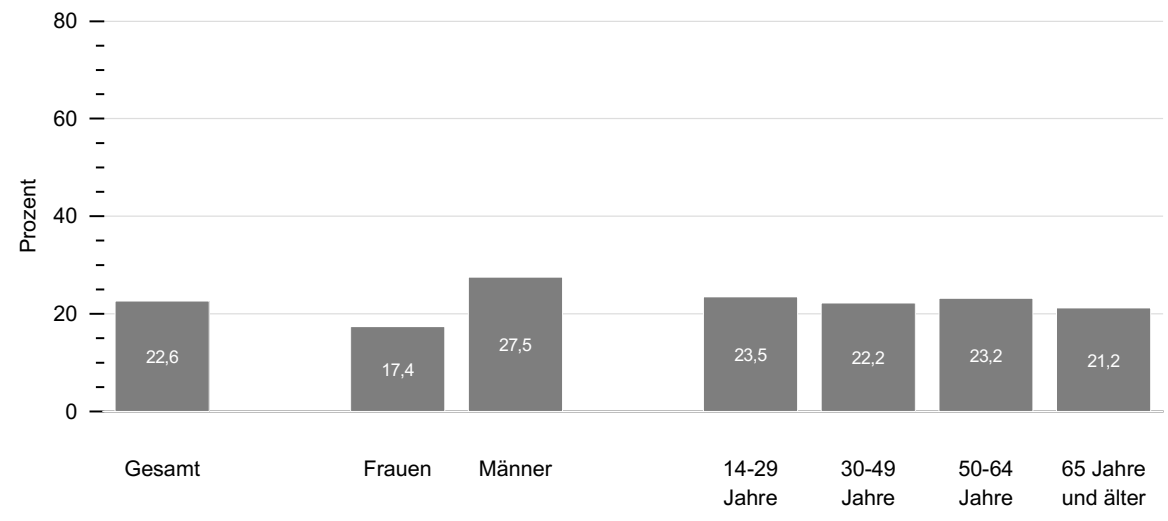

b) nach Bildung

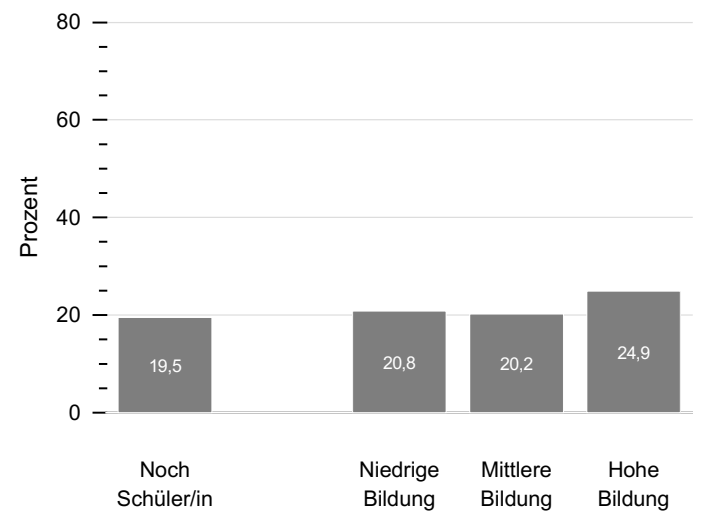

Quelle: FWS 2014, gewichtet, eigene Berechnungen (DZA). Basis: Alle Engagierten. Gesamt, Alter und Geschlecht ( $n=12.405)$, Bildung $(n=12.401)$. 
In Abbildung 13-2a sind die geschlechtsspezifischen Unterschiede in den Qualifikationsanforderungen nach Altersgruppen dargestellt. Der Befund, dass Frauen seltener eine Tätigkeit ausüben, für die eine spezielle Aus- oder Weiterbildung notwendig ist, ist in allen Altersgruppen zu beobachten. Dabei ist der Geschlechterunterschied in der Altersgruppe der 14- bis 29-Jährigen jedoch am geringsten. Für 25,8 Prozent der engagierten Männer und für 20,9 Prozent der engagierten Frauen in dieser Altersgruppe ist eine spezifische Qualifikation für ihre freiwillige Tätigkeit erforderlich. Die größten Geschlechterdifferenzen sind dagegen in der Altersgruppe der 30- bis 49-Jährigen zu finden. Während hier nur 15,1 Prozent der engagierten Frauen eine spezielle Aus- oder Weiterbildung vorweisen müssen, ist der Anteil bei den Männern dieser Altersgruppe mit 29,5 Prozent nahezu doppelt so hoch.

Abbildung 13-2: Anteile der Engagierten, für deren freiwillige Tätigkeit eine spezifische Aus- oder Weiterbildung erforderlich ist, 2014, a) nach Alter und Geschlecht, b) nach Bildung und Geschlecht

a) nach Alter und Geschlecht

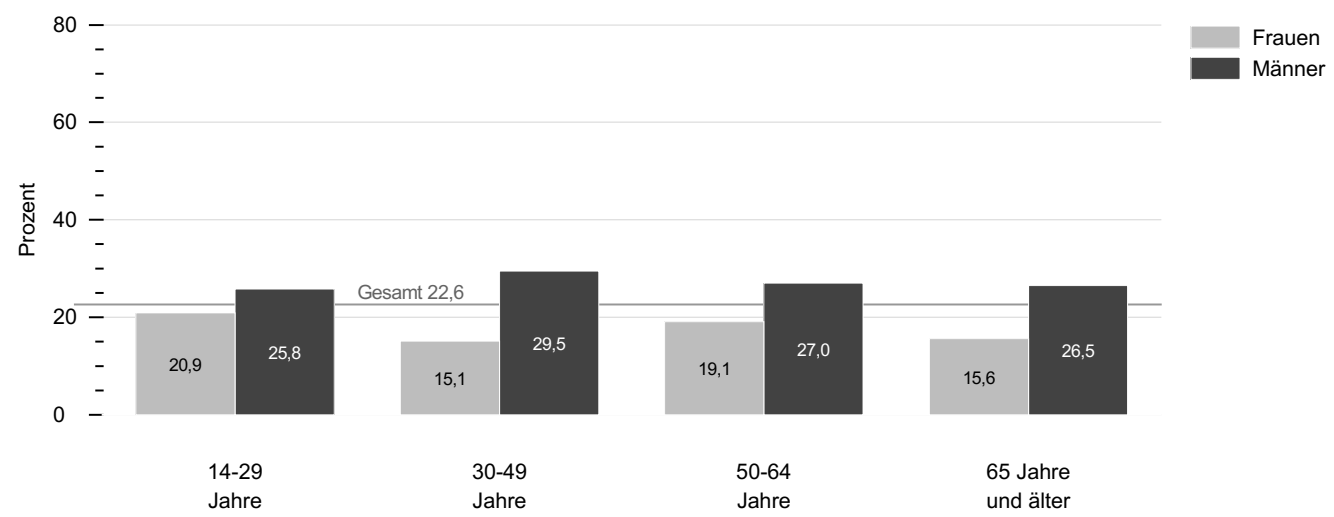

b) nach Bildung und Geschlecht

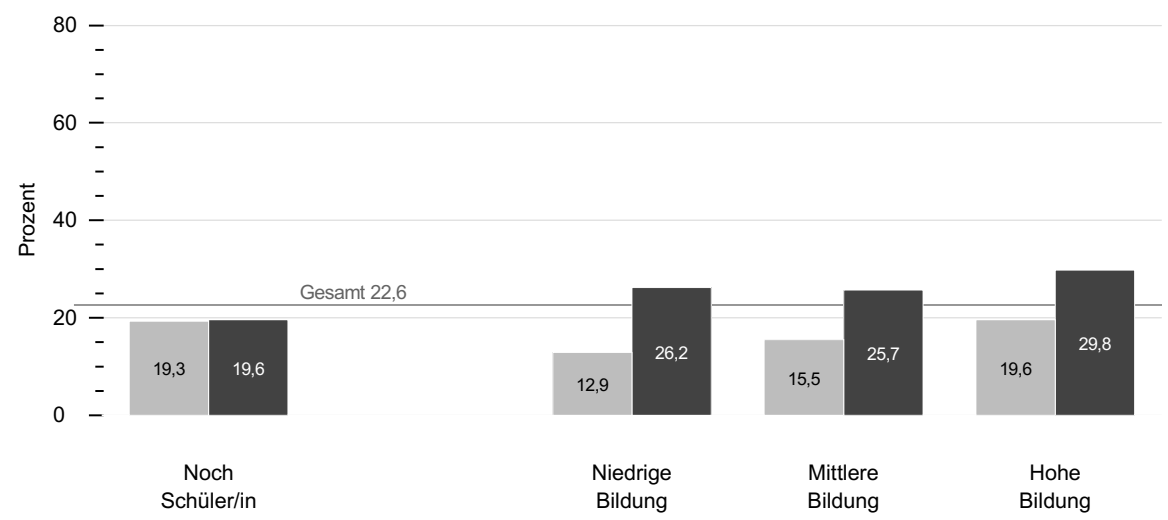

Quelle: FWS 2014, gewichtet, eigene Berechnungen (DZA). Basis: Alle Engagierten. Alter und Geschlecht $(n=12.405)$, Bildung und Geschlecht $(n=12.401)$. 
Signifikante Unterschiede in den Qualifikationsanforderungen zwischen den Geschlechtern sind auch in den verschiedenen Bildungsgruppen zu erkennen (Abbildung 13-2b). Die einzige Ausnahme bildet die Gruppe der Schülerinnen und Schüler, in der keine signifikanten Unterschiede zwischen Männern (19,6 Prozent) und Frauen (19,3 Prozent) zu beobachten sind.

Darüber hinaus unterscheiden sich die Qualifikationsanforderungen deutlich zwischen den Engagementbereichen (Abbildung 13-3). Insbe- sondere bei freiwilligen Tätigkeiten im Bereich der Unfall- oder Rettungsdienste ist der Nachweis einer Aus- oder Weiterbildung deutlich häufiger notwendig. In diesem Bereich müssen 72,3 Prozent der Engagierten eine spezifische Ausbildung oder Weiterbildung vorweisen. In den Bereichen Schule und Kindergarten, Politik und politische Interessenvertretung sowie Freizeit und Geselligkeit sind diese Anteile deutlich niedriger.

\section{Abbildung 13-3: Anteile der Engagierten, für deren freiwillige Tätigkeit eine spezifische Aus- oder Weiterbildung erforderlich ist, 2014, nach gesellschaftlichen Bereichen}

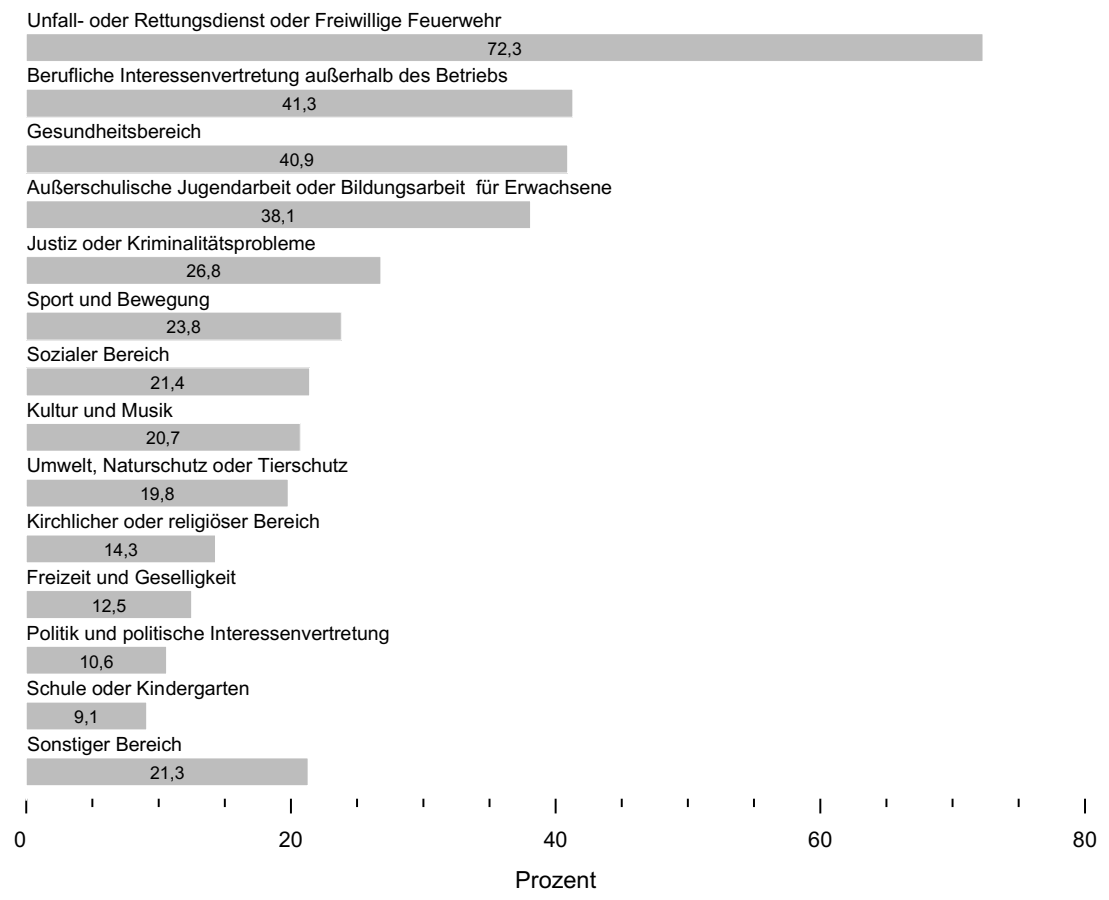

Quelle: FWS 2014, gewichtet, eigene Berechnungen (DZA). Basis: Alle Engagierten ( $n=12.315$ ). 
In allen Bereichen sind Geschlechterunterschiede hinsichtlich der Notwendigkeit einer spezifischen Aus- oder Weiterbildung zu finden. In Abbildung 13-4 zeigt sich, dass in fast allen der vierzehn Engagementbereiche mehr Männer als Frauen eine Tätigkeit ausüben, für die eine spezifische Ausbildung oder Weiterbildung erforderlich ist.

\section{Abbildung 13-4: Anteile der Engagierten, für deren freiwillige Tätigkeit eine spezifische Aus- oder Weiterbildung erforderlich ist, 2014, nach gesellschaftlichen Bereichen und Geschlecht}

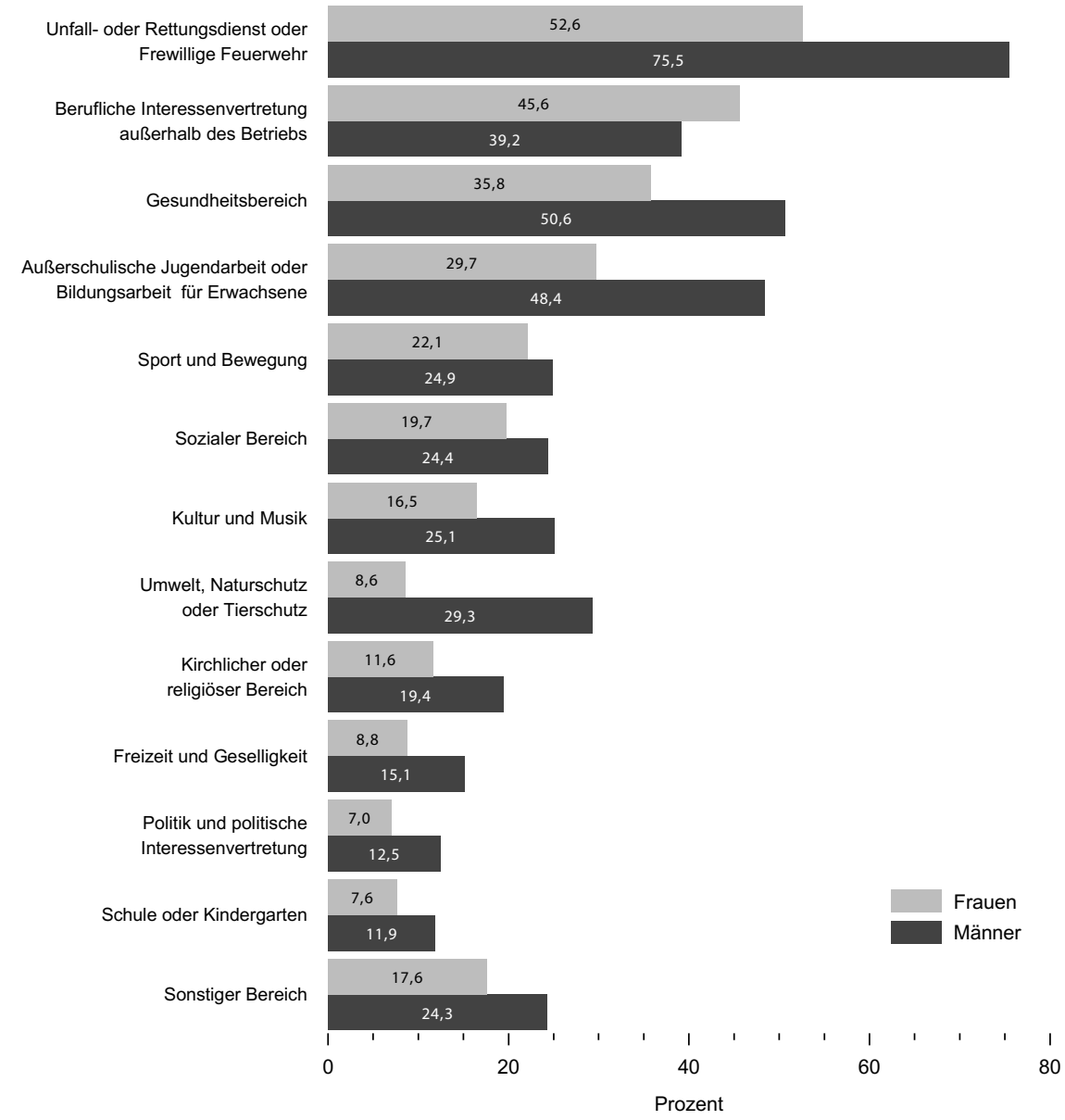

Quelle: FWS 2014, gewichtet, eigene Berechnungen (DZA). Basis: Alle Engagierten ( $n=12.241)$.

Für den Bereich ,Justiz oder Kriminalitätsprobleme' werden aufgrund geringer Fallzahlen $(\mathrm{n}<50)$ keine Ergebnisbalken differenziert nach Geschlecht dargestellt.

Der größte Unterschied ist im Bereich der Rettungsdienste zu finden, in dem 75,5 Prozent der engagierten Männer und 52,6 Prozent der engagierten Frauen angeben, dass sie für ihre Tä- tigkeit eine spezifische Aus- oder Weiterbildung benötigen. Die einzige Ausnahme ist der Bereich der beruflichen Interessenvertretung. Hier ist der Anteil der engagierten Frauen (45,6 Prozent), für 
deren freiwillige Tätigkeit eine spezifische Qualifikation erforderlich ist, höher als der Anteil der Männer (39,2 Prozent). Die Geschlechterunterschiede sind möglicherweise auf unterschiedliche Tätigkeiten und Funktionen zurückzuführen, die Frauen und Männer im Engagement ausüben. Dies kann auf unterschiedliche Präferenzen, aber auch auf Zugangsbarrieren für spezifische Funktionen zurückzuführen sein. So haben Frauen in allen gesellschaftlichen Bereichen des freiwilligen Engagements seltener eine Leitungsfunktion als Männer (siehe Kapitel 11). Aber auch wenn Frauen eine Leitungsfunktion haben, berichten sie seltener über die Notwendigkeit einer speziellen Aus- oder Weiterbildung (Abbildung 13-5). Circa ein Drittel der Männer, die eine Leitungsfunktion ausüben, berichtet über spezielle Qualifikationsanforderungen, aber nur circa ein Viertel der Frauen in Leitungsfunktionen - die Voraussetzungen für die Leitungsfunktionen, die von Frauen oder Männern ausgeübt werden, sind also offensichtlich unterschiedlich.

\section{Abbildung 13-5: Anteile der Engagierten, für deren freiwillige Tätigkeit eine spezifische Aus- oder Weiterbildung erforderlich ist, 2014, nach Leitungsfunktion und Geschlecht}

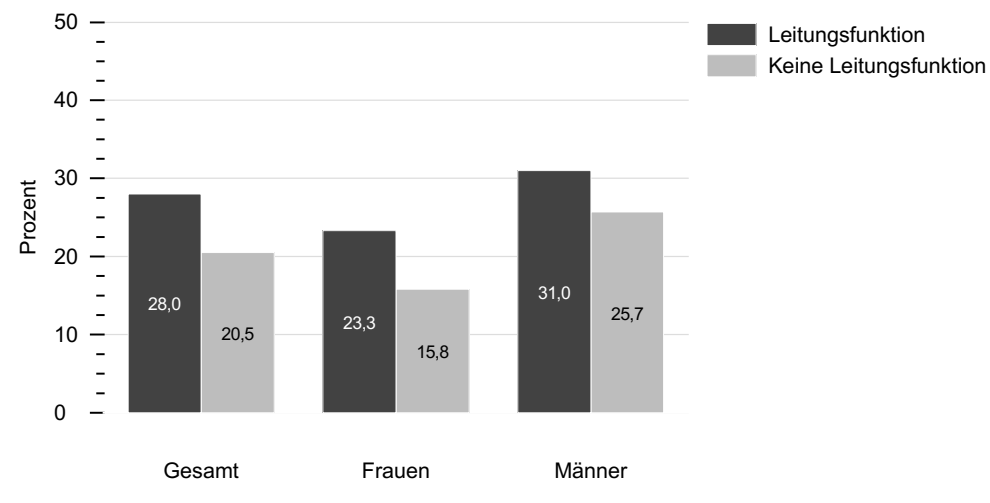

Quelle: FWS 2014, gewichtet, eigene Berechnungen (DZA). Basis: Alle Engagierten ( $n=12.391$ ).

\subsection{Weiterbildungsteilnahme im Engagement}

Im letzten Abschnitt wurde gezeigt, dass für viele freiwillige Tätigkeiten eine spezifische Aus- oder Weiterbildung erforderlich ist und dass Männer öfter eine solche Tätigkeit ausüben als Frauen. Weiterbildungsmaßnahmen können aber auch begleitend zum freiwilligen Engagement wahrgenommen werden. Im Folgenden wird ergänzend betrachtet, wie verbreitet die Teilnahme an Weiterbildungen im freiwilligen Engagement ist und wie sich die Teilnahmequoten zwischen verschiedenen Personengruppen unterscheiden.
In Abbildung 13-6a ist zu erkennen, dass 40,5 Prozent aller Engagierten schon im Rahmen ihres Engagements an einer Weiterbildung teilgenommen haben. 11,0 Prozent haben einmal und 29,5 Prozent bereits mehrmals an einer Weiterbildung teilgenommen. Dabei zeigt sich ein statistisch signifikanter Geschlechterunterschied: Frauen nehmen seltener an Weiterbildungen im Rahmen ihres freiwilligen Engagements teil als Männer. Insgesamt haben 43,6 Prozent der engagierten Männer mindestens eine Weiterbildung im Rahmen ihrer freiwilligen Tätigkeit besucht, 
aber nur 37,3 Prozent der Frauen. Darüber hinaus haben engagierte Männer zu größeren Anteilen bereits mehrmals an einer Weiterbildung teilgenommen als Frauen (32,7 Prozent versus 26,2 Prozent).

Nach Altersgruppen differenziert sind ebenfalls signifikante Unterschiede $\mathrm{zu}$ beobachten. In den beiden jüngeren Altersgruppen (14- bis 29- sowie 30- bis 49-Jährige) ist der Anteil derer, die schon Weiterbildungen absolviert haben, niedriger (37,3 Prozent und 35,3 Prozent) als in den beiden älteren Altersgruppen (46,2 Prozent für die 50- bis 64-Jährigen und 45,9 Prozent für Personen ab 65 Jahren). Dabei sind alle Altersgruppenunterschiede statistisch signifikant mit Ausnahme der Differenz zwischen den beiden ältesten Gruppen. Die niedrigeren Quoten bei den jüngeren Altersgruppen könnten darauf zurückzuführen sein, dass diese aufgrund ihres jüngeren Alters und der im Durchschnitt kürzeren biografischen Dauer des Engagements noch nicht so viele Gelegenheiten hatten, an Weiterbildungen teilzunehmen. Es könnten aber auch Unterschiede in den freiwilligen Tätigkeiten zum Tragen kommen. So üben Jüngere beispielsweise seltener beratende Tätigkeiten aus und haben auch seltener eine Leitungsfunktion (siehe Kapitel 11).
In Abbildung 13-6b ist die Teilnahme an Weiterbildungen für die verschiedenen Bildungsgruppen dargestellt. Es gibt keinen statistisch signifikanten Unterschied zwischen Personen mit niedriger, mittlerer oder hoher Bildung; bei allen drei Gruppen beträgt der Anteil derer, die im Rahmen ihres freiwilligen Engagements an einer oder mehreren Weiterbildungen teilgenommen haben, etwa 41 Prozent. Eine Ausnahme bilden die Schülerinnen und Schüler, bei denen der Anteil der Engagierten, die schon an Weiterbildungen teilgenommen haben, signifikant niedriger ist (31,7 Prozent). Auch der Anteil der engagierten Schülerinnen und Schüler, die bereits mehrmals an einer Weiterbildung teilgenommen haben, ist signifikant geringer (14,4 Prozent) als in den anderen Bildungsgruppen (circa 30 Prozent). Diese Unterschiede lassen sich in ähnlicher Weise interpretieren wie die berichteten Alterseffekte. Schülerinnen und Schüler sind in der Regel jünger als Personen mit abgeschlossener Schulbildung und werden daher bislang weniger Gelegenheiten zur Weiterbildung gehabt haben. Darüber hinaus können auch hier Unterschiede in den Tätigkeiten, die im freiwilligen Engagement ausgeübt werden, eine Rolle spielen (siehe Kapitel 11). 
Abbildung 13-6: Anteile der Engagierten, die an einer Weiterbildung teilgenommen haben, 2014, a) gesamt, nach Geschlecht und nach Alter, b) nach Bildung

a) gesamt, nach Geschlecht und nach Alter

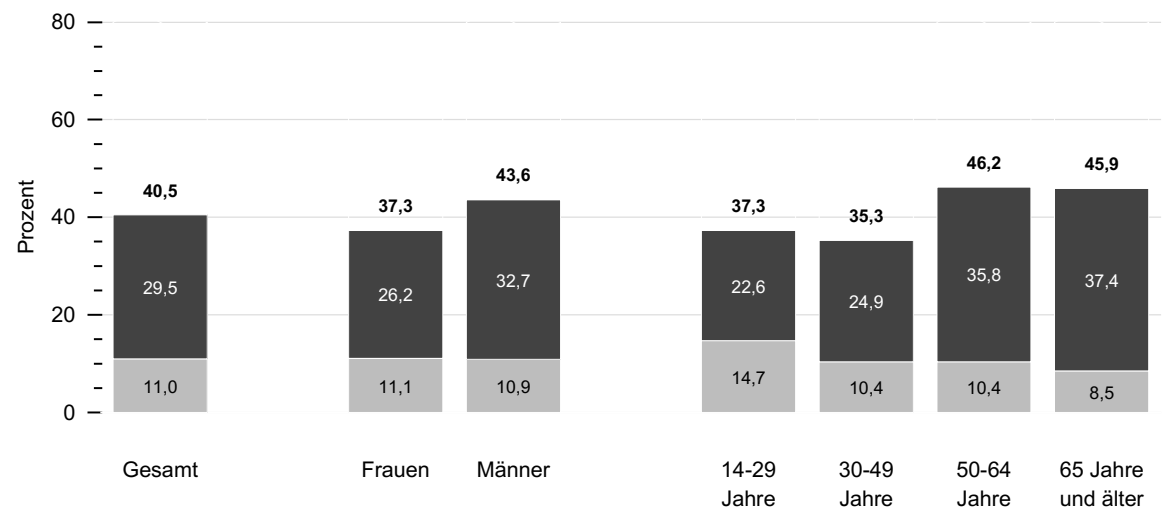

\section{b) nach Bildung}

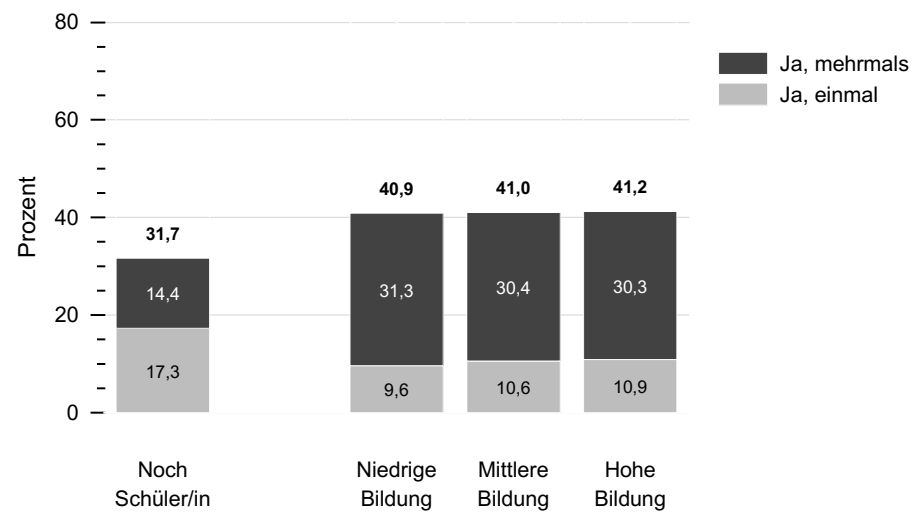

Quelle: FWS 2014, gewichtet, eigene Berechnungen (DZA). Basis: Alle Engagierten. Gesamt, Alter und Geschlecht ( $n=12.423)$, Bildung $(n=12.419)$. 
Abbildung 13-7: Anteile der Engagierten, die an einer Weiterbildung teilgenommen haben, 2014, a) nach Alter und Geschlecht, b) nach Bildung und Geschlecht

a) nach Alter und Geschlecht

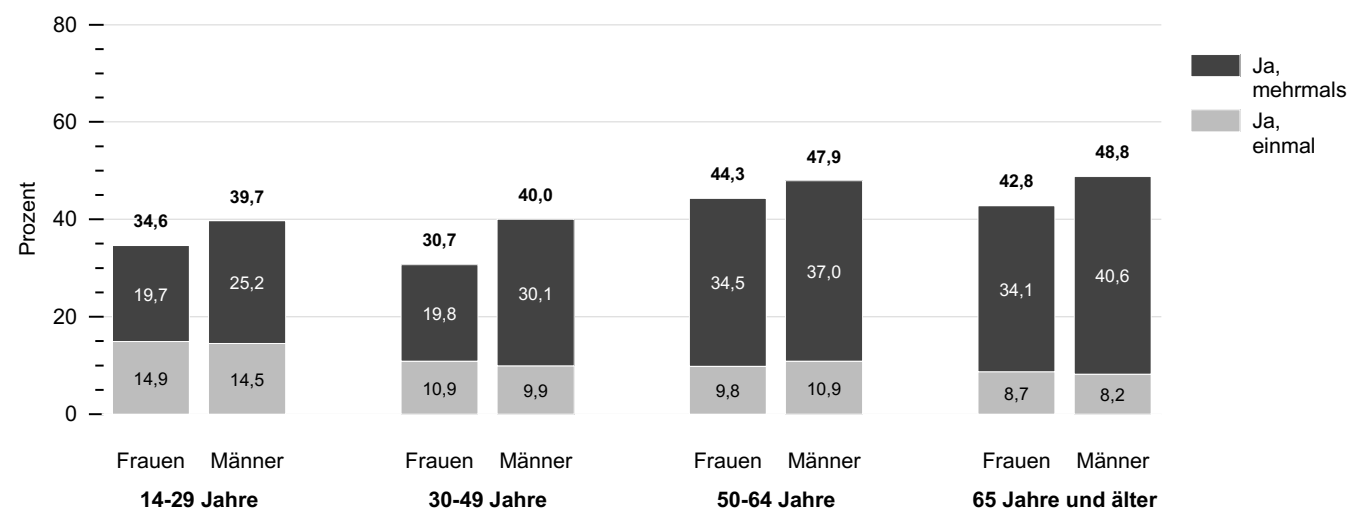

b) nach Bildung und Geschlecht

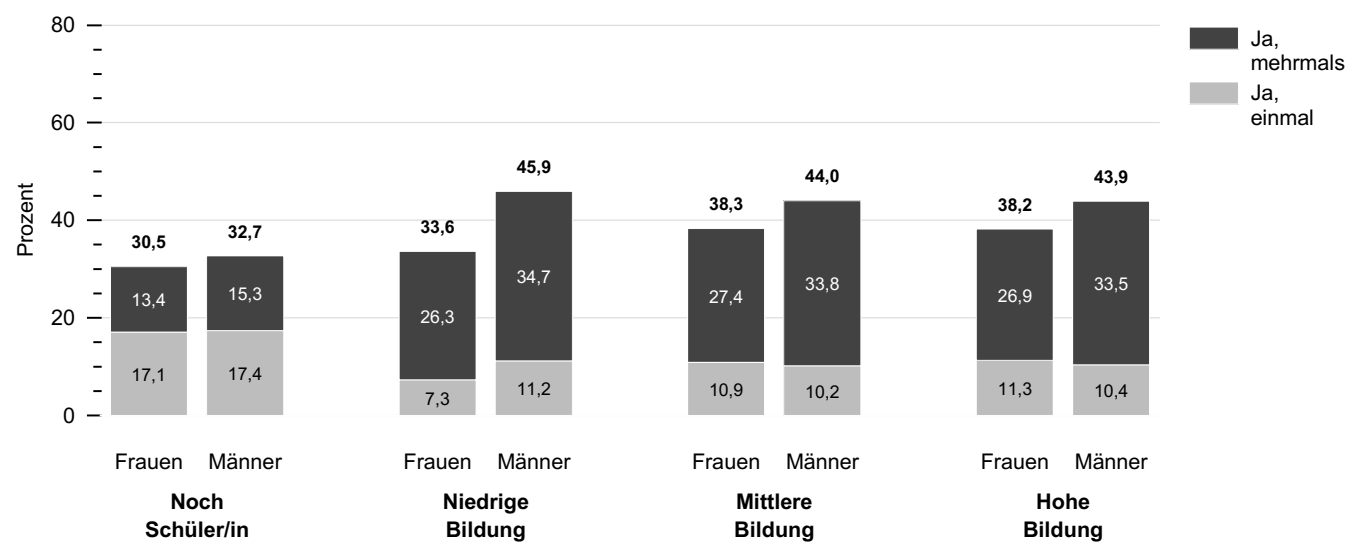

Quelle: FWS 2014, gewichtet, eigene Berechnungen (DZA). Basis: Alle Engagierten. Alter und Geschlecht $(n=12.423)$, Bildung und Geschlecht $(n=12.419)$.

In Abbildung 13-7a ist zu sehen, dass Geschlechterunterschiede in Bezug auf die Teilnahme an Weiterbildungen in allen Altersgruppen $\mathrm{zu}$ erkennen sind. Nur die Unterschiede für die Altersgruppe der 14- bis 29-Jährigen sind nicht statistisch signifikant. Die höchste Differenz zwischen Männer und Frauen finden wir in der Altersgruppe der 30- bis 49-Jährigen (30,7 Prozent für Frauen und 40,0 Prozent für Männer).
Die Geschlechterunterschiede in der Weiterbildungsteilnahme variieren zwischen den verschiedenen Bildungsgruppen (Abbildung 13-7b). Die Differenzen sind mit Ausnahme der Schülerinnen und Schüler für alle Gruppen statistisch signifikant. Der größte Unterschied zwischen Frauen und Männern ist in der niedrigsten Bildungsgruppe $\mathrm{zu}$ finden. In dieser Gruppe haben 45,9 Prozent der Männer, aber nur 33,6 
Prozent der Frauen schon an Weiterbildungen im Engagement teilgenommen.

In der Abbildung 13-8 wird die Teilnahme an Weiterbildungen nach Bereichen dargestellt. Es sind Parallelen zur Abbildung 13-3 zu erkennen. In den Bereichen, in denen öfter über besondere Qualifikationsanforderungen berichtet wird, ist die Teilnahme an Weiterbildungen höher. Das ist der Fall für die Bereiche Unfall- oder Rettungsdienst, berufliche Interessenvertretung, den
Gesundheitsbereich sowie den Bereich Jugendarbeit/Erwachsenenbildung. Darüber hinaus gibt es andere Bereiche, in denen seltener über spezielle Qualifikationsanforderungen berichtet wird, aber die Teilnahme an Aus- oder Weiterbildung relativ häufig ist. Das ist der Fall für die Bereiche Politik und politische Interessenvertretung, Justiz oder Kriminalitätsprobleme, für den sozialen Bereich sowie den Bereich Kirche und Religion.

\section{Abbildung 13-8: Anteile der Engagierten, die an einer Weiterbildung teilgenommen haben, 2014, nach gesellschaftlichen Bereichen}

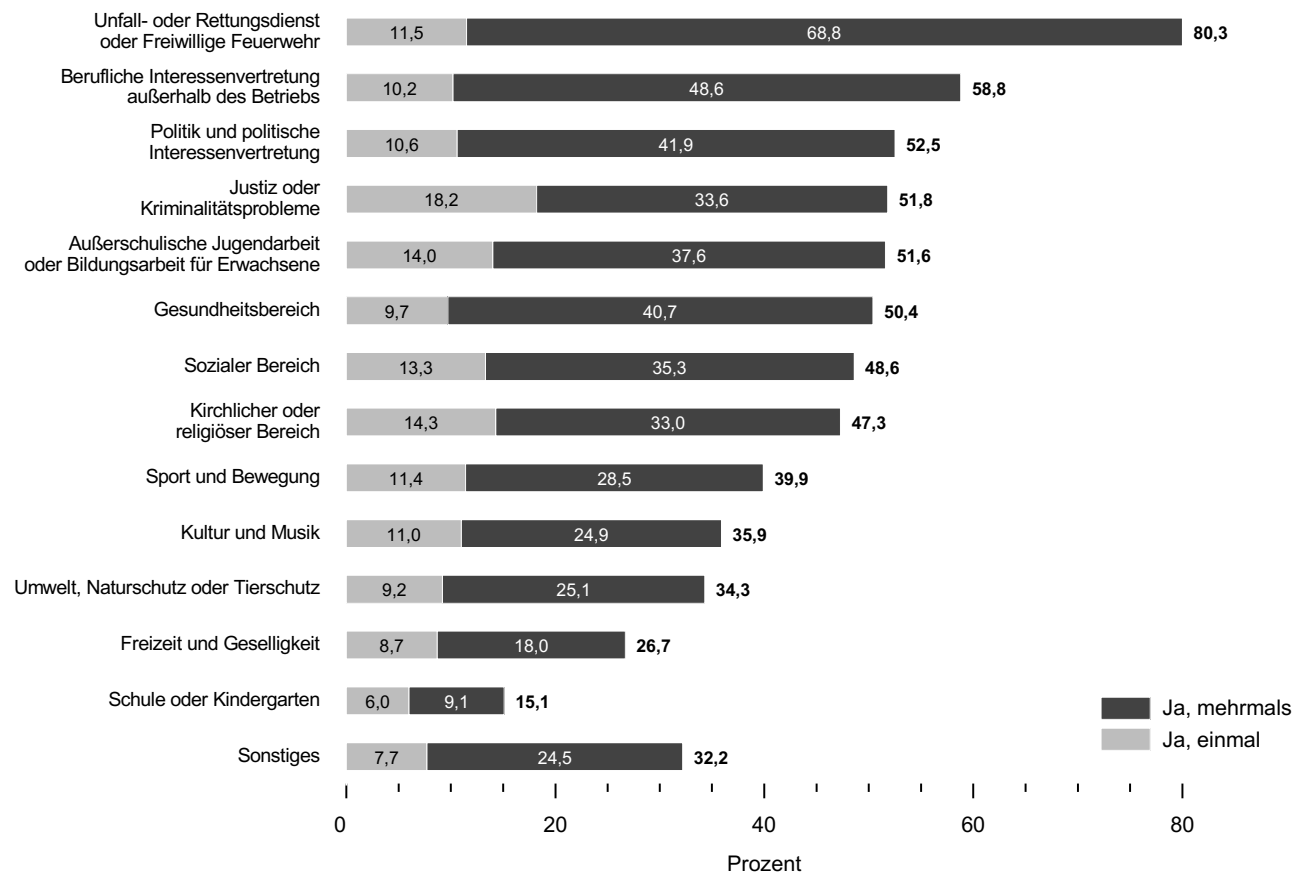

Quelle: FWS 2014, gewichtet, eigene Berechnungen (DZA). Basis: Alle Engagierten ( $n=12.333$ ). 


\subsection{Erwerb von Fähigkeiten im Engagement}

Fähigkeiten und Kenntnisse können nicht nur im Rahmen von Weiterbildungsmaßnahmen erworben werden, sondern auch während der Ausübung der freiwilligen Tätigkeit selbst. Im Folgenden wird dargestellt, welche Kenntnisse und Fähigkeiten die Engagierten im Engagement erworben haben und inwieweit diese Kenntnisse ihnen im schulischen und beruflichen Bereich von Nutzen sind. Gezeigt wird auch, ob Frauen und Männer, unterschiedliche Altersgruppen beziehungsweise unterschiedlich gebildete Menschen verschieden von den im Rahmen des Engagements erworbenen Kenntnissen profitieren.

Alle Engagierten wurden im Freiwilligensurvey 2014 gefragt, ob sie im Rahmen ihrer freiwilligen Tätigkeit Fachkenntnisse, soziale Fähigkeiten (zum Beispiel Teamfähigkeit, Zuhören können) oder persönliche Fähigkeiten (zum Beispiel Selbstständigkeit, Zeitmanagement oder Arbeitstechniken) erworben haben. In Abbildung 13-9a wird gezeigt, welche Kenntnisse oder Fähigkeiten im Engagement meistens erlernt werden und ob es Geschlechter- und Altersunterschiede bei den erworbenen Fähigkeiten gibt.

Insgesamt ist der Erwerb von Kenntnissen im Engagement weit verbreitet. Der Anteil der Engagierten, die keine Fähigkeiten genannt haben, beträgt nur 14,7 Prozent. Am häufigsten geben Engagierte an, soziale Fähigkeiten erworben $\mathrm{zu}$ haben (73,7 Prozent). Darüber hinaus haben 57,7 Prozent der Engagierten persönliche Fähigkeiten in ihrem Engagement erworben und 52,8 Prozent Fachkenntnisse.

Dabei sind Geschlechterunterschiede zu beobachten. Männer berichten signifikant häufiger über erworbene Fachkenntnisse (59,5 Prozent) als Frauen (45,7 Prozent). Die Geschlechterunterschiede in den Anteilen der Engagierten, die berichten, soziale beziehungsweise persönliche Fähigkeiten erworben zu haben, sind zwar gering, aber statistisch signifikant. Dieses Ergebnis kann zum Teil auf die verschiedenen Funktionen und Tätigkeiten von Frauen und Männern im freiwilligen Engagement zurückzuführen sein (siehe Kapitel 11).

Darüber hinaus lassen sich Altersunterschiede feststellen. Es zeigt sich, dass Jüngere in besonderem Maße Kenntnisse und Fähigkeiten im Engagement erwerben. Besonders häufig wird von den 14- bis 29-Jährigen, im Vergleich zu den anderen Altersgruppen, der Erwerb von sozialen und persönlichen Fähigkeiten genannt. Keine dieser Fähigkeiten dazu gewonnen zu haben, geben in dieser Altersgruppe nur 5,3 Prozent der Engagierten an.

In Abbildung 13-9b sind Bildungsunterschiede in Bezug auf im Engagement erworbene Kenntnisse oder Fähigkeiten dargestellt. Zu bemerken ist, dass Schülerinnen und Schüler, auch wenn es sonst keine großen Unterschiede zwischen den Bildungsgruppen gibt, öfter als andere berichten, soziale (86,7 Prozent) und persönliche Fähigkeiten (78,7 Prozent) zu erlangen. Dieses Ergebnis deckt sich mit den Altersgruppenunterschieden und belegt die Relevanz, die freiwilliges Engagement gerade bei jüngeren Menschen für den Erwerb von sozialen und persönlichen Kompetenzen hat. 
Abbildung 13-9: Anteile der Engagierten, die berichten Fähigkeiten im Rahmen ihrer Tätigkeit erworben zu haben, 2014, a) gesamt, nach Geschlecht und nach Alter, b) nach Bildung

a) gesamt, nach Geschlecht und nach Alter

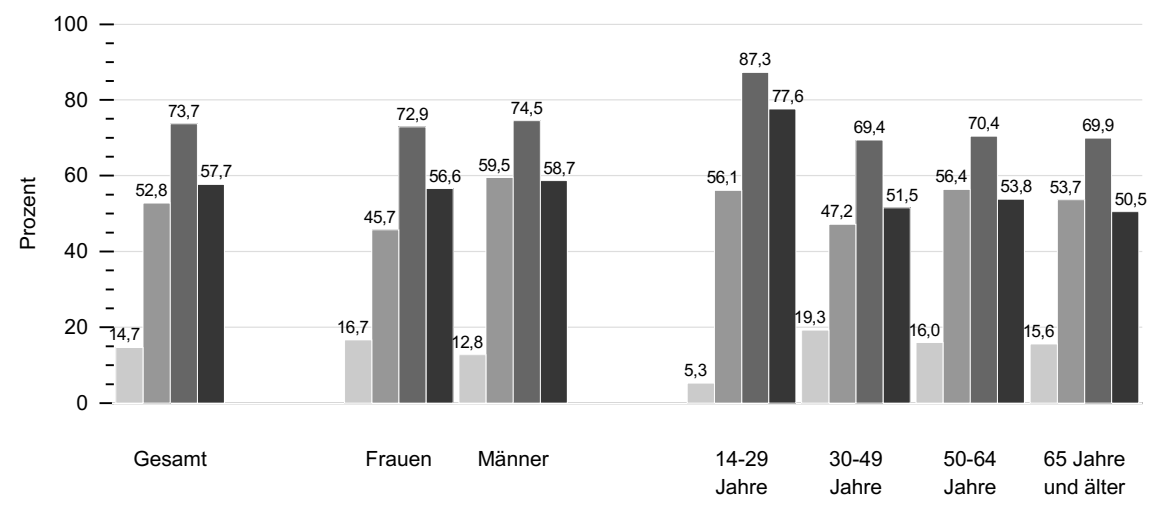

b) nach Bildung

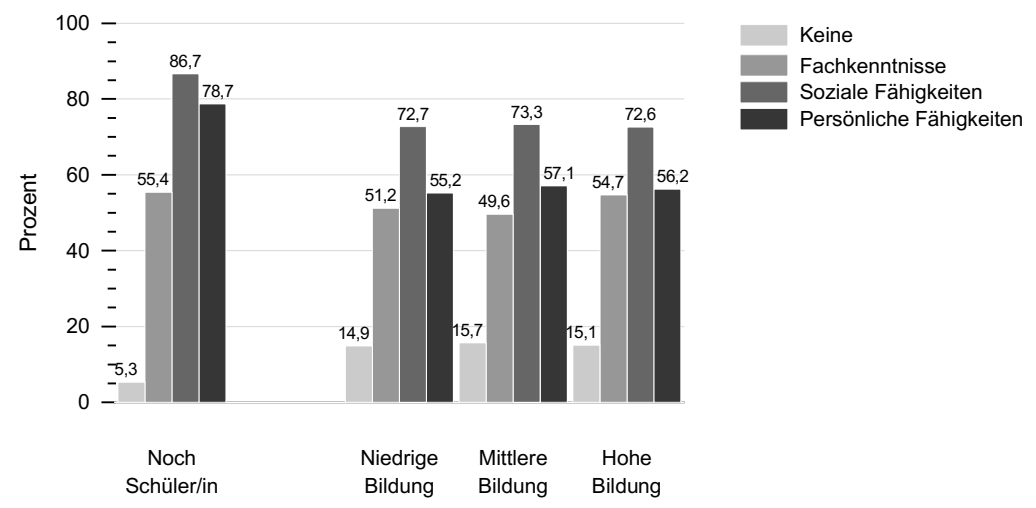

Quelle: FWS 2014, gewichtet, eigene Berechnungen (DZA). Basis: Alle Engagierten. Gesamt, Alter und Geschlecht ( $n=12.382-12.399)$. 
Abbildung 13-10 zeigt die Geschlechterunterschiede in den im Engagement erworbenen Fähigkeiten für die verschiedenen Altersgruppen. In allen Altersgruppen außer den 14- bis 29-Jährigen ist hinsichtlich der erworbenen Fachkenntnisse ein signifikanter Geschlechterunterschied festzustellen. In dieser jüngsten Altersgruppe be- richten 53,6 Prozent der Frauen und 58,4 Prozent der Männer über erworbene Fachkenntnisse. In der Altersgruppe der 30- bis 49-Jährigen beträgt der Anteil der Frauen, die Fachkenntnisse erworben haben, nur 39,1 Prozent, gegenüber 55,6 Prozent bei den Männern dieser Altersgruppe.

\section{Abbildung 13-10: Anteile der Engagierten, die berichten Fähigkeiten im Rahmen ihrer Tätigkeit erworben zu haben, 2014, nach Geschlecht und Alter}

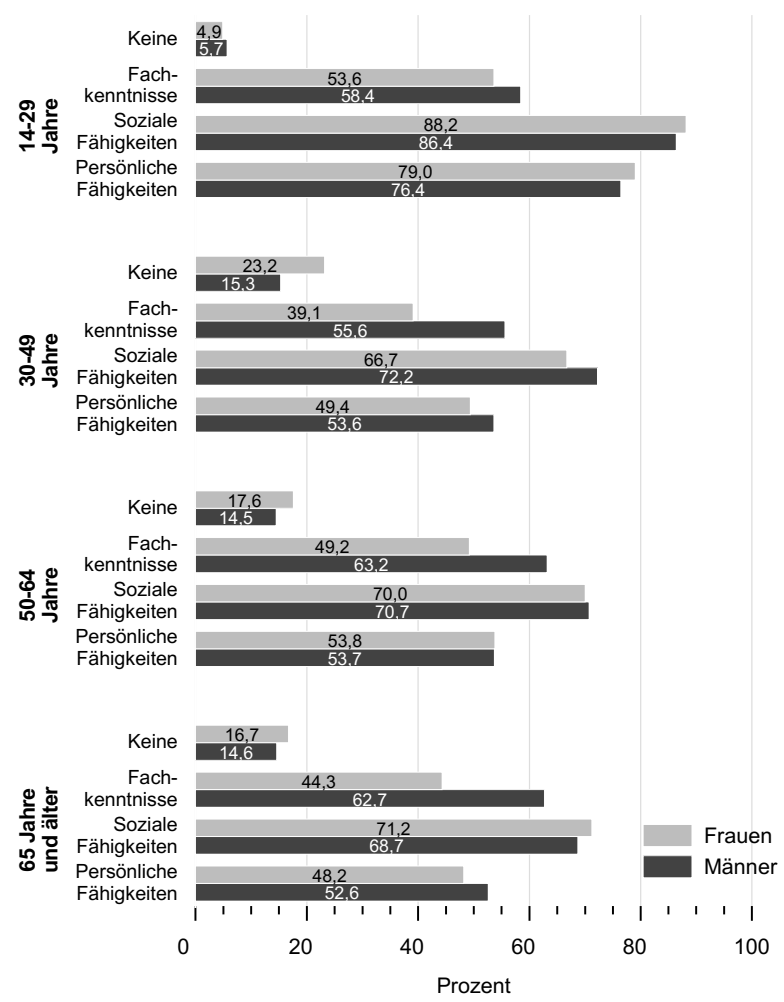

Quelle: FWS 2014, gewichtet, eigene Berechnungen (DZA). Basis: Alle Engagierten ( $n=12.382-12.399$ ). 


\subsection{Subjektiver Nutzen der im Engagement erworbenen Fähigkeiten und Kenntnisse}

Im vorangegangenen Abschnitt wurde gezeigt, dass ein Großteil der Engagierten durch die freiwillige Tätigkeit verschiedene Fähigkeiten und Kenntnisse erworben hat. Diese Kompetenzen können nicht nur für die freiwillige ausgeübte Tätigkeit nützlich sein, sondern auch darüber hinaus. Im Folgenden wird der Frage nachgegangen, inwiefern die erworbenen Kenntnisse nützlich für das berufliche Leben beziehungsweise die Schule, eine Ausbildung oder ein Studium sind.

In den Abbildungen 13-11 bis 13-13 sind die Anteile von Personen dargestellt, die über die Nützlichkeit der Fähigkeiten und Kenntnisse für (1) Schule, Ausbildung, oder Studium, für (2) den beruflichen Wiedereinstieg und für (3) die berufliche Tätigkeit berichten. In Abbildung 13-11 sehen wir zunächst, dass 84,6 Prozent der
Engagierten, die noch zur Schule gehen oder sich in einem Studium oder in Ausbildung befinden und die in ihrer freiwilligen Tätigkeit Kenntnisse oder Fähigkeiten erworben haben, berichten, dass diese nützlich für Schule, Studium oder Ausbildung sind. Wir haben im letzten Abschnitt gesehen, dass Schülerinnen und Schüler im Vergleich zu den anderen Bildungsgruppen am häufigsten Fähigkeiten und Kenntnisse in ihrem Engagement erwerben. Diese Kenntnisse sind für einen Großteil auch nützlich für die Schule. Die Differenz zwischen Frauen (86,4 Prozent) und Männern (82,9 Prozent) ist dabei statistisch signifikant, Frauen geben also etwas häufiger an, dass sie die erworbenen Fähigkeiten beziehungsweise Kenntnisse für die Schule, ihre Ausbildung oder ihr Studium nutzen können.

\section{Abbildung 13-11: Anteile der Engagierten, die berichten, die im Engagement erworbenen Fähigkeiten seien nützlich für Schule, Studium oder Ausbildung, 2014, gesamt und nach Geschlecht}

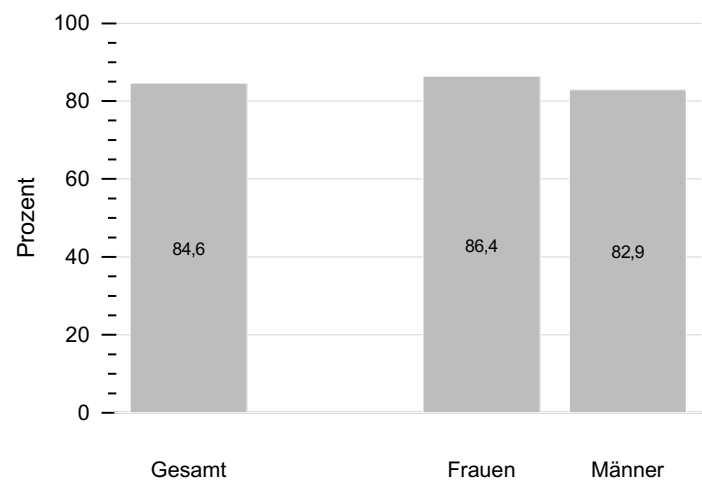

Quelle: FWS 2014, gewichtet, eigene Berechnungen (DZA). Basis: Alle Engagierten in Schule, Ausbildung, Studium, die berichten Fähigkeiten erworben zu haben $(n=1.478)$.

Darüber hinaus berichten 59,2 Prozent der Engagierten (nur Personen in Arbeitslosigkeit, Mutterschutz/Elternzeit oder Umschulung/ Weiterbildung sowie Personen, die seit weniger als einem Jahr erwerbstätig sind), dass die im Engagement erworbenen Fähigkeiten für ihren beruflichen Wiedereinstieg nützlich sind (Abbildung 13-12a). Geschlechterunterschiede und 
Altersgruppenunterschiede sind im diesen Fall nicht statistisch signifikant. Im Bildungsvergleich zeigt sich, dass Engagierte mit höherer Bildung ihre im Engagement erworbenen Fähigkeiten am häufigsten beim beruflichen Wiedereinstieg nutzen können (Abbildung 13-12b)

Abbildung 13-12: Anteile der Engagierten, die berichten, die im Engagement erworbenen Fähigkeiten seien nützlich für den beruflichen Wiedereinstieg, 2014, a) gesamt, nach Geschlecht und nach Alter, b) nach Bildung

a) gesamt, nach Geschlecht und nach Alter

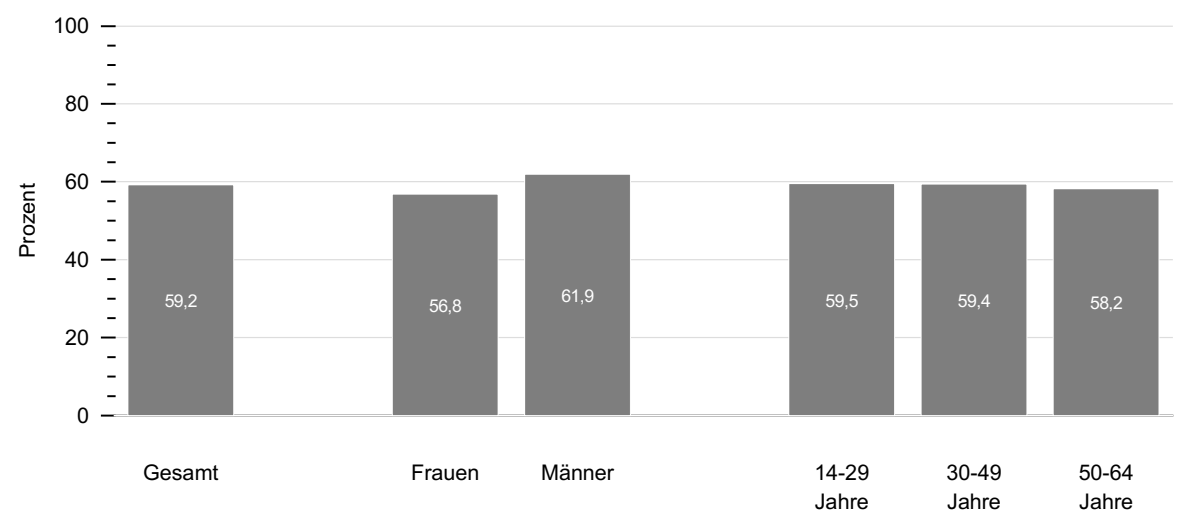

b) nach Bildung

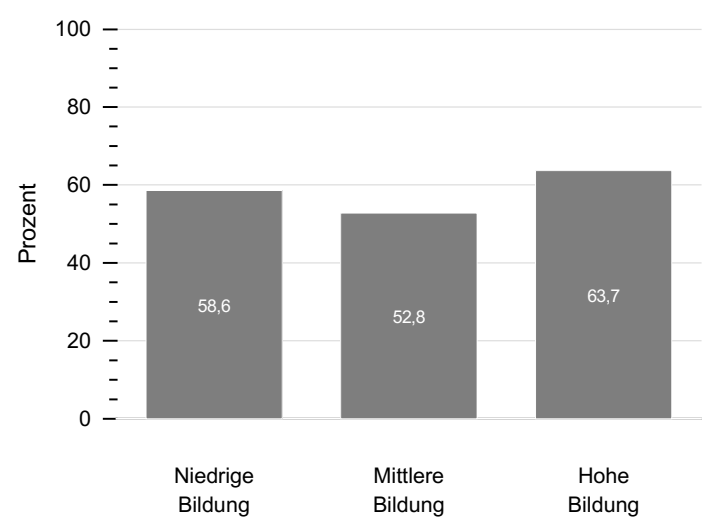

Quelle: FWS 2014, gewichtet, eigene Berechnungen (DZA). Basis: Alle Engagierten, die arbeitslos, in Mutterschutz, Elternzeit, in Umschulung/Weiterbildung oder seit weniger als einem Jahr erwerbstätig sind, und berichten, Fähigkeiten erworben zu haben $(n=573)$. 
Abbildung 13-13: Anteile der Engagierten, die berichten, die im Engagement erworbenen Fähigkeiten seien nützlich für die berufliche Tätigkeit, 2014, a) gesamt, nach Geschlecht und nach Alter, b) nach Bildung

a) gesamt, nach Geschlecht und nach Alter

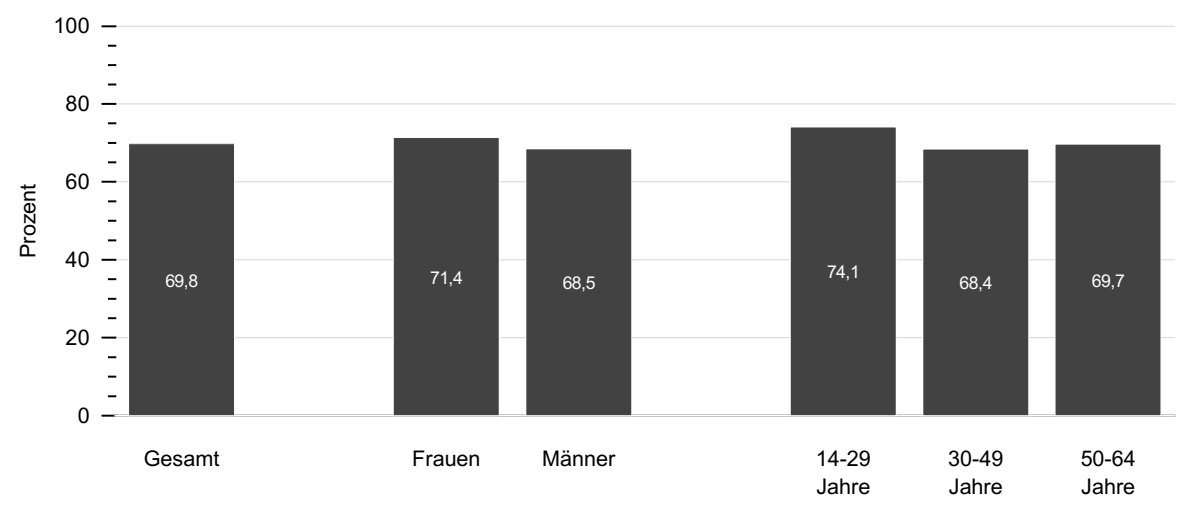

b) nach Bildung

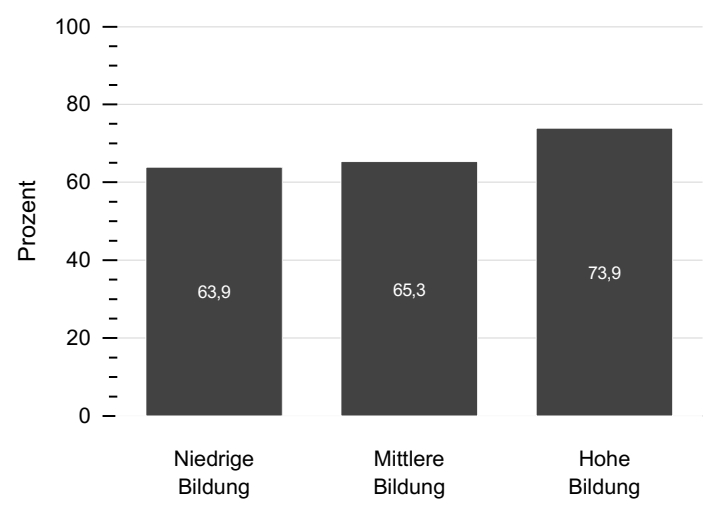

Quelle: FWS 2014, gewichtet, eigene Berechnungen (DZA). Basis: Alle erwerbstätige Engagierten, die berichten Fähigkeiten, erworben zu haben. Gesamt, Alter und Geschlecht $(n=5.508)$, Bildung $(n=5.507)$. Die älteste Gruppe (65 Jahre und älter) wird aufgrund der geringen Anzahl von Erwerbstätigen in diesem Alter nicht ausgewiesen.

Insgesamt 69,8 Prozent der erwerbstätigen Engagierten berichten, dass die erworbenen Kenntnisse nützlich für ihre berufliche Tätigkeit sind (Abbildung 13-13a). Dabei sind signifikante Geschlechterunterschiede zu beobachten. Mehr Frauen (71,4 Prozent) als Männer (68,5 Prozent) berichten, dass die erworbenen Kenntnisse für ihre berufliche Tätigkeit von Nutzen sind. Auch zwischen den Altersgruppen sind signifikante Unterschiede zu finden. Engagierte der jüngs- ten Altersgruppe (14- bis 29-Jährige) berichten anteilig öfter (74,1 Prozent) als die anderen Altersgruppen, dass sie einen Nutzen für ihre berufliche Tätigkeit aus den erworbenen Fähigkeiten und Kenntnissen ziehen können. Auch in diesen Altersgruppen ist jedoch der Prozentsatz derer, die den Kenntnisgewinn für ihre Erwerbstätigkeit nutzen können, hoch $(68,4$ und 69,7 Prozent). Insgesamt zeigen die Ergebnisse, dass die Kenntnisse und Fähigkeiten, die Personen im 
Rahmen ihrer freiwilligen Tätigkeiten erwerben, nicht nur für das Engagement selbst, sondern auch für die berufliche Sphäre von Bedeutung sind. Nach Bildung ist zu beobachten, dass En- gagierte mit höherer Bildung öfter von den im Engagement erworbenen Fähigkeiten profitieren (sowohl in Hinsicht auf den beruflichen Wiederanstieg als auch für die berufliche Tätigkeit).

\subsection{Fazit}

Fast ein Viertel der Engagierten übt eine freiwillige Tätigkeit aus, für die eine spezifische Aus- oder Weiterbildung erforderlich ist. Dabei benötigen deutlich mehr Männer als Frauen eine spezifische Qualifikation für ihre freiwillige Tätigkeit. Darüber hinaus bestehen große Unterschiede bei den Qualifikationsanforderungen zwischen den jeweiligen gesellschaftlichen Bereichen des Engagements. Im Unfall- und Rettungsdienstbereich benötigen die Engagierten am häufigsten eine spezielle Aus- oder Weiterbildung.

Zwei Fünftel aller Engagierten haben im Rahmen ihres Engagements schon einmal oder mehrmals an einer Weiterbildung teilgenommen. Auch hier zeigen sich geschlechtsspezifische Differenzen: Frauen nehmen anteilig deutlich seltener als Männer an Weiterbildungen im Rahmen ihres Engagements teil. Dies könnte zum einen daran liegen, dass Frauen, wie gezeigt, eher Tätigkeiten ausüben, für die keine spezifische Qualifikation notwendig ist. Ein weiterer Grund könnte sein, dass bestimmte, notwendige Weiterbildungen als Barriere für Frauen wirken, wenn Frauen sich zwar für die gleiche Tätigkeit interessieren, aber zum Beispiel aufgrund von familiären Verpflichtungen nicht die Flexibilität haben, eine Weiterbildung dafür zu machen. Wenn dies der Fall ist, ist zum einen zu überlegen, wie diese Weiterbildungsangebote organisiert werden können, sodass auch Frauen die gleichen Zugangschancen dazu haben. Zum anderen können auch Maßnahmen, die zu einer ausgeglicheneren Arbeitsteilung zwischen den Geschlechtern im familiären und beruflichen Bereich beitragen, die Teilnahmemöglichkeiten für Frauen verbessern.
Keine Unterschiede hinsichtlich der Weiterbildungsteilnahme zeigen sich dagegen zwischen Personen mit niedriger, mittlerer und hoher Schulbildung. Die Annahme, dass Weiterbildungsangebote im Engagement hauptsächlich den ohnehin schon Hochgebildeten zukommen und somit soziale Ungleichheiten im Engagement befördern können, bestätigt sich also nicht. Eine deutliche Bildungsselektion findet bereits beim Zugang zum freiwilligen Engagement statt: Mit steigender Schulbildung sind höhere Raten des freiwilligen Engagements $\mathrm{zu}$ beobachten (siehe Kapitel 3); wenn sich Personen mit niedrigerer Bildung aber erst einmal engagieren, nehmen sie mit ähnlicher Wahrscheinlichkeit an Weiterbildungsangeboten teil wie Personen mit mittlerer oder höherer Bildung.

Im freiwilligen Engagement können unterschiedliche Kenntnisse und Fähigkeiten erworben werden. Am häufigsten geben Engagierte an, soziale Fähigkeiten erworben zu haben, gefolgt von persönlichen Fähigkeiten und Fachkenntnissen. Auch hier zeigen sich Geschlechterdifferenzen; Männer geben deutlich häufiger als Frauen an, Fachkenntnisse erworben zu haben. Unterschiedliche Tätigkeiten und Funktionen, die Männer und Frauen im Engagement ausüben, könnten hierfür eine Erklärung sein - ebenso wie die unterschiedlich verbreitete Teilnahme an Weiterbildungen.

Schülerinnen und Schüler berichten öfter als die anderen Engagierten, durch ihr Engagement Fähigkeiten und Kenntnisse zu erwerben. Darüber hinaus zeigt sich, dass die von Schülerinnen und Schülern, Auszubildenden und Studierenden erworbenen Kenntnisse nach ihren Angaben häufig nützlich für Schule, Ausbildung oder Stu- 
dium sind. Diese Ergebnisse weisen in dieselbe Richtung wie die Studie von Düx et al. (2009), in der auf Basis qualitativer Interviews gezeigt wird, dass Jugendliche durch das freiwillige Engagement nachhaltige und exklusive Lernerfahrungen machen können. Diese Studie zeigt auch, dass Jugendliche neben sozialen und persönlichkeitsbezogenen Eigenschaften und Fähigkeiten über das freiwillige Engagement insbesondere auch Organisations-, Leitungs-, Team- und Gremienkompetenzen entwickeln. Engagement hat also im jungen Alter positive Auswirkungen auf Schule, Ausbildung und Studium. Die Förderung des Engagements junger Menschen erscheint vor diesem Hintergrund besonders bedeutsam.

Es zeigt sich allerdings, dass das Engagement auch in späteren Lebensphasen den Erwerb von Kenntnissen und Fähigkeiten fördert und dadurch positive Auswirkungen auf das berufliche Leben haben kann. Freiwilliges Engagement kann also für den Kompetenzerwerb in allen Lebensphasen bedeutsam sein. Neben den positiven Effekten des Engagements auf die Gesundheit (siehe Kapitel 17) und die bessere soziale Einbettung der engagierten Personen (siehe Kapitel 19), erwerben die engagierten Frauen und
Männer Kompetenzen, die für ihr berufliches und soziales Leben wichtig sind.

Allerdings zeigt sich auch, dass Engagierte mit höherer Bildung am häufigsten angeben, von den erworbenen Fähigkeiten zu profitieren: Sie berichten häufiger als Engagierte mit niedriger oder mittlerer Bildung, dass die im Engagement erworbenen Fähigkeiten für den beruflichen Wiedereinstieg oder für die berufliche Tätigkeit von Nutzen sind. Es gibt also keine Bildungsselektion bei der Teilnahme an Weiterbildungen im Engagement, aber Hochgebildete profitieren mehr davon. Denkbar ist, dass Personen mit höherer Bildung andere und arbeitsmarktkompatiblere Fähigkeiten im freiwilligen Engagement erwerben als Personen mit niedrigerer Bildung. Möglicherweise schaffen es Höhergebildete aber auch besser, die im Engagement erworbenen Kenntnisse auf andere Lebensbereiche zu übertragen. Bei Personen mit niedriger Bildung könnten Benachteiligungsmechanismen auf dem Arbeitsmarkt diesem Transfer entgegenstehen. Die im Engagement erworbenen Kenntnisse und Fähigkeiten verringern die bestehenden Chancenungleichheiten von höher und niedriger gebildeten Personen auf dem Arbeitsmarkt also nicht.

\section{Literatur}

Düx, W. (2006). „Aber so richtig für das Leben lernt man eher bei der freiwilligen Arbeit“. Zum Kompetenzgewinn Jugendlicher im freiwilligen Engagement. In: T. Rauschenbach, W. Düx \& E. Sass (Hrsg.) Informelles Lernen im Jugendalter. Vernachlässigte Dimensionen der Bildungsdebatte (S. 205-240). Weinheim: Juventa.

Düx, W., Prein, G., Sass, E., \& Tully, C. (2009). Kompetenzerwerb im freiwilligen Engagement. Eine empirische Studie zum informellen Lernen im Jugendalter. Wiesbaden: VS Verlag für Sozialwissenschaften.

Enquete-Kommission ,Zukunft des Bürgerschaftlichen Engagements' (2002). Bericht der Enquete-Kommission ,Zukunft des Bürgerschaftlichen Engagements'. Bürgerschaftliches Engagement: auf dem Weg in eine zukunftsfähige Bürgergesellschaft (Bundestagsdrucksache 14/8900). Berlin: Deutscher Bundestag.

Gensicke, T. (2015). Freiwilliges Engagement in Deutschland: Freiwilligensurvey 2009. Wiesbaden: Springer VS.

Hartnuß, B., \& Kegel, T. (2011). Qualifizierung. In: T. Olk \& B. Hartnuß (Hrsg.) Handbuch Bürgerschaftliches Engagement (S. 623-633). Weinheim: Beltz Juventa.

Jakob, G. (2013). Qualifizierung als Auftrag einer modernen Engagementförderung. Qualifiziert engagiert - Engagiert qualifiziert. Stärkung bürgerschaftlichen Engagements durch Fort- und Weiterbildungsangebote. Wiesbaden: Hessische Landesregierung.

Leipold, B. (2012). Lebenslanges Lernen und Bildung im Alter. Stuttgart: Kohlhammer. 
Mildenberger, G. (2012). Qualifizierung und Anreiz für das Engagement im Konzept der Sozialen Investitionen. In: H. Anheier, A. Schröer \& V. Then (Hrsg.) Soziale Investitionen. Interdisziplinäre Perspektiven (S. 167-180). Wiesbaden: VS Verlag für Sozialwissenschaften.

Naumann, D., \& Romeu Gordo, L. (2010). Gesellschaftliche Partizipation: Erwerbstätigkeit, Ehrenamt und Bildung. In: A. Motel-Klingebiel, S. Wurm \& C. Tesch-Römer (Hrsg.) Altern im Wandel. Befunde des Deutschen Alterssurveys (DEAS) (S. 118-141). Stuttgart: Kohlhammer.

Prein, G., Sass, E., \& Züchner, I. (2009). Lernen im freiwilligen Engagement und gesellschaftliche Partizipation. Ein empirischer Versuch zur Erklärung politischen Handelns. Zeitschrift für Erziehungswissenschaft, 12(3), 529-547.

Reich-Claassen, J., \& Tippelt, R. (2013). Erwachsenen- und Weiterbildungspolitik - Zur Notwendigkeit der Berücksichtigung der mittleren und späteren Lebensphasen in der Bildungspolitik. In: M. Hüther \& G. Naegele (Hrsg.) Demografiepolitik. Herausforderungen und Handlungsfelder (S. 179-199). Wiesbaden: Springer VS.

Simonson, J., Hagen, C., Vogel, C., \& Motel-Klingebiel, A. (2013). Ungleichheit sozialer Teilhabe im Alter. Zeitschrift für Gerontologie und Geriatrie, 46(5), 410-416.

Open Access Dieses Kapitel wird unter der Creative Commons Namensnennung 4.0 International Lizenz (http://creativecommons.org/licenses/by/4.0/deed.de) veröffentlicht, welche die Nutzung, Vervielfältigung, Bearbeitung, Verbreitung und Wiedergabe in jeglichem Medium und Format erlaubt, sofern Sie den/die ursprünglichen Autor(en) und die Quelle ordnungsgemäß nennen, einen Link zur Creative Commons Lizenz beifügen und angeben, ob Änderungen vorgenommen wurden.

Die in diesem Kapitel enthaltenen Bilder und sonstiges Drittmaterial unterliegen ebenfalls der genannten Creative Commons Lizenz, sofern sich aus der Abbildungslegende nichts anderes ergibt. Sofern das betreffende Material nicht unter der genannten Creative Commons Lizenz steht und die betreffende Handlung nicht nach gesetzlichen Vorschriften erlaubt ist, ist für die oben aufgeführten Weiterverwendungen des Materials die Einwilligung des jeweiligen Rechteinhabers einzuholen. 